\title{
APPLIANCE RECOGNITION USING A DENSITY- BASED CLUSTERING APPROACH WITH MULTIPLE GRANULARITIES
}

\author{
Chun-Wei Yen, Yu-Lin Ke,Sheng-Ta Chen, Yi-Chieh Pai, Hung-Chieh \\ Wei, and Wei-Guang Teng \\ Department of Engineering Science, \\ National Cheng Kung University, Tainan, Taiwan
}

\begin{abstract}
Electricity may not be economically stored as other forms of energy such that it would be in short supply during the peak time. In view of this difficulty, most power suppliers encourage their customers to adopt time-of-use rate plans. Consequently, it is essential for a user to be able to perceive the real-time information of power consumption. With the advancement of Internet of Things technologies, smart sockets are becoming a commodity to manage power consumption in a household. However, current smart sockets merely present the total electricity consumption rather than the individual consumption of household appliances. In this work, we thus design the capability of appliance recognition and implement this feature into a smart socket so that we can identify the power consumption of each appliance respectively. The proposed recursive DBSCAN approach realizes the recognition task without prior knowledge of new appliances and shows a feasible result in our experimental studies.
\end{abstract}

\section{KEYWORDS}

Appliance Recognition, Data Clustering, Smart Socket

\section{INTRODUCTION}

Reducing fossils dependence, tackling global warming, strengthening energy security have gained significant attention in recent years. Electricity, unlike other forms of energy (e.g., coal, gas, and oil,) may not be economically stored and must be generated on demand. In realistic environments like the electricity market, the electricity real-time balance is an essential task of the power system [1]. It is necessary for the power suppliers to guide the users to adjust the load from the demand side to help them solve the problem of insufficient power supply during the peak time [2]. The most common and effective method is the time-of-use pricing [3], which means power supplier reflects the difference in power supply cost at different times and provides the user with the correct price information to promote system load balancing and improve the effective use of power by sharply separating the peak price. For the user, instantly grasp the load curve of all electrical appliances can proactively shift the load of using the appliance to avoid high rate electricity price time. On the other hand, knowing the inefficiency of old electrical appliances with instant load curve can help users making choices and plans for the use of electrical appliances. Accordingly, smart sockets are produced for equipping in a smart grid environment to improve the monitoring of electricity consumption [4].

Smart sockets on the market cannot be widely used in a typical environment. The main reason is high installation cost since one smart socket can only measure one single electrical device. The 
existing smart sockets are typically installed as a form of adapters which causes users inconvenient to make use of it. To address these critical issues, we propose to design and implement an embedded smart socket. Specifically, the designed smart socket is appropriate to be used in the common living environment without changing usage habit.

Applying the technology of appliance recognition in smart socket is an appropriate method to further reduce installation cost. However, to realize an embedded smart socket in a practical application scenario, we find out that it is unsuitable to adopt the existing appliance recognition approaches. In addition, depending on the scenario of a new appliance plug into our smart sockets, no pre-training method for appliance recognition is required. Based on the above reasons, we adopt the density-based clustering approach. In this paper, we proposed a recursive DBSCANbased method to realize the embedded smart socket, applying with a user interface and learning from user feedback can increase the efficiency and effectiveness of power analyzation in the living environment scenario.

The rest of this paper is organized as follows. Related works are generally explored in Sec.2. Details of our approach are presented in Sec.3. Experimental results and the corresponding discussion are illustrated in Sec.4. Finally, Sec.5 concludes this paper.

\section{RELATED WORKS}

Resulting from the ubiquitous home network has gained widespread attention due to its seamless integration into everyday life [5], smart sockets which are responsible for recording the electricity so that user can be widely used to manage their energy usage in time. A smart socket makes use of sensors, actuators and wireless transceivers to collect the information of pricing and the user preferences and to optimize home energy consumption [6]. Users can keep track of their electrical appliances through a user-friendly interface to improve price-aware decision-making since energy consumption can be collected easily and monitored. Not only providing the household with energy feedback on a single appliance but also allowing a better understanding of computing the relative contribution of each appliance to the global consumption [7].

Nevertheless, the smart sockets on the market only can measure the single electrical device. This is a very inefficient way to achieve a truly smart home if setting an appliance to pair with a smart socket. In order to overcome the limitation in our scenario, applying the technology of appliance recognition is an appropriate method because that electrical loads usually exhibit a unique energy consumption pattern and the pattern data can be acquired from the smart socket. Consumption pattern which can be termed as "load of appliance signatures" [8] enables the disaggregation algorithms to discern and recognize appliance operations from the aggregated load measurements. Appliance recognition is highly dependent on the load signature since most appliances can be distinguished from each other with having different unique power signatures. Power signatures usually can be divided into steady-state ones and transient ones [8]. Common steady-state signatures are extracted and used in general appliance recognition algorithms like the real (active) power $(\mathrm{P})$ and the reactive power $(\mathrm{Q})$. The voltage $(\mathrm{V})$ and current $(\mathrm{I})$ are frequently used as well for easily being distinguished [9]. Transient signatures like turn-on transient instantaneous power which is generated when the appliance is operated [10], start-up current transients and high frequency sampling of voltage noise [8] can also serve as reliable metrics for load recognition. A cepstrum-smoothing-based load disaggregation method is proposed to effectively deal with the simultaneous on/off events of multiple appliances since multiple appliances can be turned on/off simultaneously in reality situation [11].

Many prior works of recognizing the electrical appliance using these signatures are developed. Researchers have tried to classify the appliances and construct the taxonomy of the appliances by 
employing a hierarchical clustering method [12]. Same with classification method, it has been proposed that a characterization method of using both in the frequency and temporal domain [13] and that using neural networks, support vector machines, k-means, mean-shift, and silhouette classifiers to achieve better effect of appliance recognition [14].Besides of these clustering approach, density-based clustering is a nonparametric approach. Clusters are identified due to their density of areas. Therefore, area of high-point density clusters can be separated by areas of low-point density, so that they can form arbitrarily shape in the data space[15].Ordering points to identify the clustering structure (OPTICS) provides a more efficient clustering approach for very high-dimensional spaces to set parameters. With this approach, clustering approaches are not limited to one global parameter setting. Instead, they create an augmented ordering of the data samples representing its density-based clustering structure. Consequently, this approach is multiskilled for automatic and interactive cluster analysis [16].Most of the research work is focus on obtaining a suitable parameter and clustering the data samples one time. However, the appliances always have load signatures that are quite different to each other. Using clustering approach only one time is not suitable for practical application scenario.

\section{Smart Socket Design And Recursive DbSCAn APPROACH}

To tackle the problems of current smart sockets on the market, we design the hardware and adopt DBSCAN to recognize appliances. However, the bottleneck in existing DBSCAN is that it cannot accurately recognize appliances which have similar features. Therefore, we implement recursive DBSCAN to solve this problem. Details of the proposed recursive DBSCAN is illustrated in later sections.

\subsection{SMART Socket DeSIGN}

The conceptual design of the smart socket for obtaining real-time information of power which would be recognized is shown in Fig.1. The socket can transmit information of power to MQTT broker through Wi-Fi, and the information transmitted is stored into the database afterward. Eventually, our system can recognize appliances by the different features of the data.

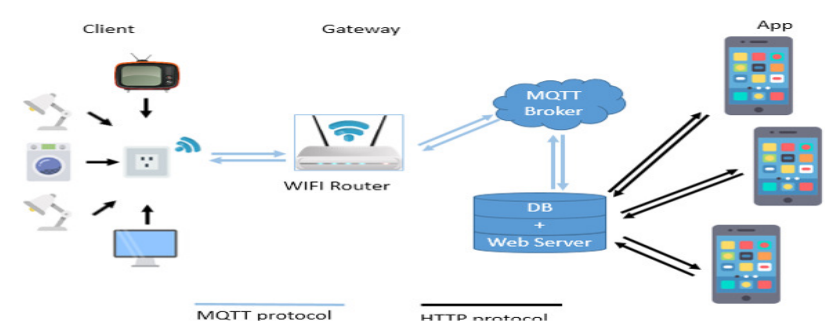

Figure 1. Conceptual design of smart socket

\subsection{Obtaining Electrical Power Characteristics}

To realize a low power consumption smart socket and save data while transmitting information on a network, we have defined three modes, which are standby mode, appliance recognition mode, and steady-state mode as shown in Fig. 2, to obtain electrical power features. 


\section{1) Standby Mode}

If there are not any appliances detected by smart socket, the smart socket may record simple information of only voltages every 30 seconds.

\section{2) RECOGNITION MODE}

If a significant change of currents is detected, the mode of our smart socket is changed into recognition mode. Detailed data of voltages $(\mathrm{V})$, currents $(\mathrm{I})$, power factor $(\mathrm{PF})$, active power $(\mathrm{P})$, reactive power $(\mathrm{Q})$ and apparent power $(\mathrm{S})$ are recorded in a high sampling frequency. Our system then starts the appliance recognition process.

\section{3) STEAdy-State Mode}

In order to reduce the storage of database and load of network bandwidth, when currents come to steady, smart socket record detailed data of voltages, currents, PF values, active power, and reactive power every 30 seconds for statistics of energy consumption.

These three modes are mainly based on detecting the change of currents. However, even if there aren't any appliances, noises caused by the electrical grid may occur. Because of this reason, we only change the mode of the smart socket when the variation of the currents is larger than the noises.

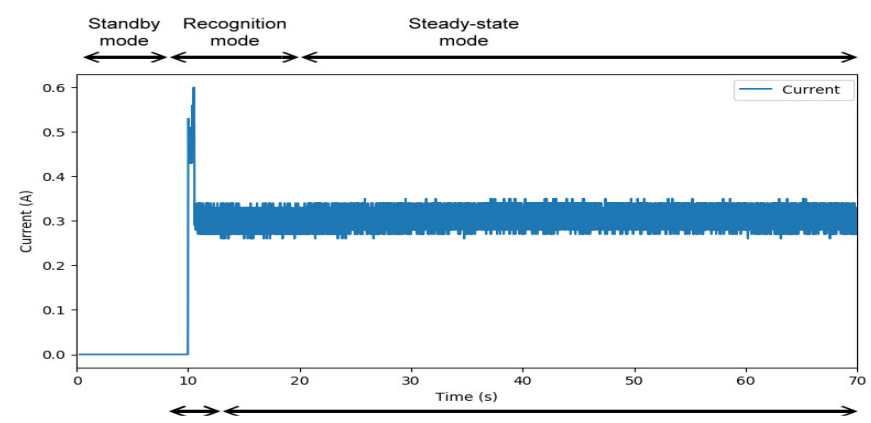

Figure 2. Three electrical power characteristics obtaining modes based on the change of currents

\subsection{APPLiAnCE RECOGNITION USING RECURSIVE DB SCAN}

In the practical application scenario, we do not have prior knowledge of the new appliances, so the classification approaches are not suitable for the appliances recognition task. For this reason, clustering is a better approach. We adopt DBSCAN as our appliances recognition method because the density-based clustering is still effective even when we do not know the number of appliances.

DBSCAN (Density-based spatial clustering of applications with noise) is a density-based algorithm which is used to discover arbitrary-shaped clusters and differentiate noise objects synchronously, it is not necessary to determine the number of clusters in the data [17]. This is the major reason why we choose DBSCAN because, in practice, the number of appliances cannot be determined at first. DBSCAN, in short, is based on distinguishing points in a high density or a low-density area. A core is determined if a given point has a high density, which means that the point has more neighbour points within the defined radius (Eps) than the defined minimum number of points (MinPts). Moreover, points are regarded as noise points if they do not have any neighbour point within Eps. 
The load signatures have an unstable performance while the appliances are just turned on during transient state as shown in Fig. 2. After the transient state, the appliances then soon turn into steady state and construct a cluster in the feature space as shown in Fig. 3. Some appliances may construct more than one clusters because of their regular changes in some load signatures. If we use the density-based clustering method for appliances recognition, we can easily cluster the load signatures without the influence of unstable load signatures in transient state

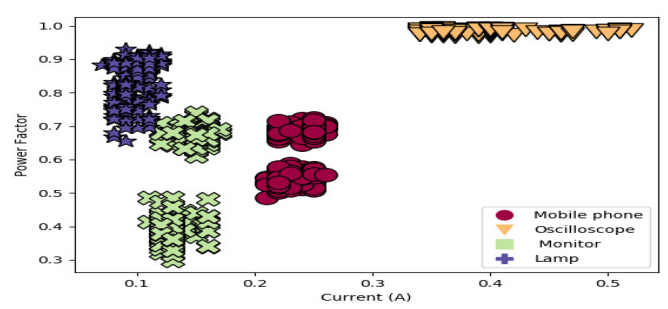

Figure 3. Load signatures construct the clusters in the feature space

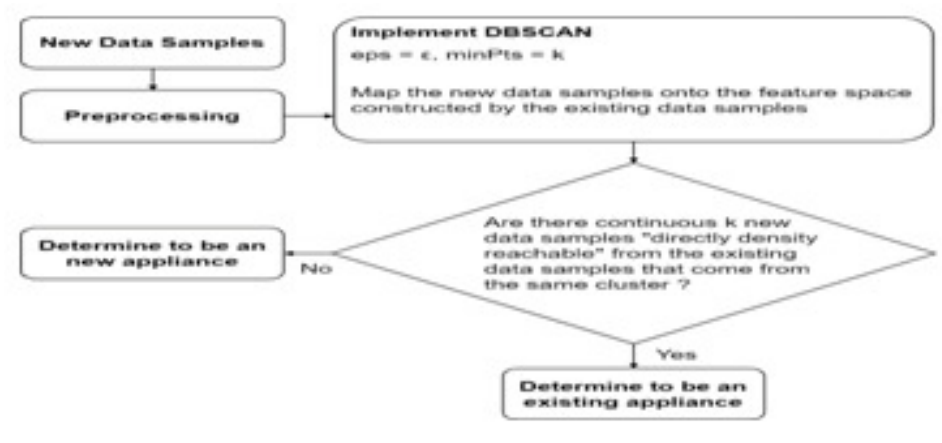

Figure 4 . The process of the appliances recognition approach

When the smart socket detects the load input, the process of our appliance recognition approach can be divided into three stages, as shown in Fig.4.These three stage sare recursively implemented with multiple granularities until successfully recognize the appliances. Some appliances with high power consumption may have the obvious load signatures difference with the appliances with low power consumption. If we only implement DBSCAN one time, it is not effective to recognize all of the appliances. Therefore, our system recursively using DBSCAN with smaller granularities for appliances with low power consumption.

We collect 6 different load features including voltage $(\mathrm{V})$, current $(\mathrm{I})$, active power $(\mathrm{P})$, reactive power $(\mathrm{Q})$, apparent power $(\mathrm{S})$ and power factor $(\mathrm{PF})$. The 6 load features have different units and number range. For example, a power factor is always a number in range 0 to 1 , but the active power does not have a specific range. To avoid the neglect of the feature with small values while implementing the clustering, we normalize the data into range 0 to 1 before adopting DBSCAN.

After pre-processing the data samples, we can start using DBSCAN and mapping the data samples onto the feature space constructed by the existing data samples in databases. Assume the minimum point of DBSCAN is $\mathrm{k}$, if there are continuous knew data samples qitoqi $+\mathrm{k}$ " directly density-reachable" from the existing data samples that come from the same clusterCi, the new data samples may belong to clusterCiand determined to be the appliance of cluster $\mathrm{Ci}$. On the other hand, if there are continuous $\mathrm{k}$ new data samplesqitoqi $\mathrm{k}$ not "directly density reachable" 
from any existing data samples, the new data samples may construct a new cluster and determined to be a new appliance.

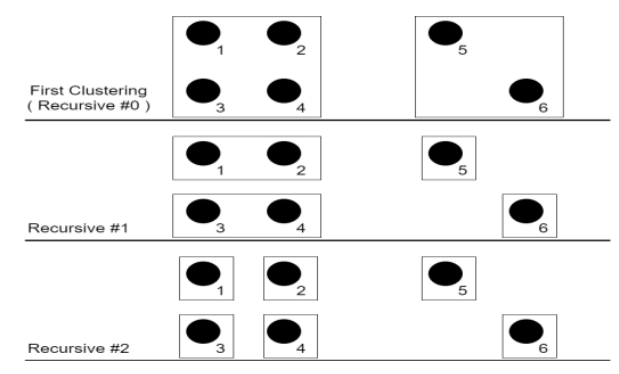

Figure 5. Concept of recursive clustering

Although almost all the appliances construct its own cluster in the feature space, different appliances are probably performing similarly sometimes. If we use DBSCAN one time, we can only easily recognize the appliances that have quite different signatures. The concept of recursive clustering is shown in Fig. 5 and each black point represents one kind of appliance. To recognize the appliances having highly similar signatures, we implement the DBSCAN recursively, scale up, and only focus on the appliances have not been recognized. As shown in Fig. 6(a), the appliances marked with red frame are quite similar in the normalized feature space and we may not able to recognize all of them by one DBSCAN process. To determine whether recursively executing the next DBSCAN or not, a user trigger mechanism is implemented in our system. When detecting the appliances are turning on, the system shows the recognition results. User can check the results and even make a correction if the system makes an incorrect recognition. In each recursion, our system retains the unrecognized appliances, normalizes the feature data again, and implement the DBSCAN with multiple granularities.

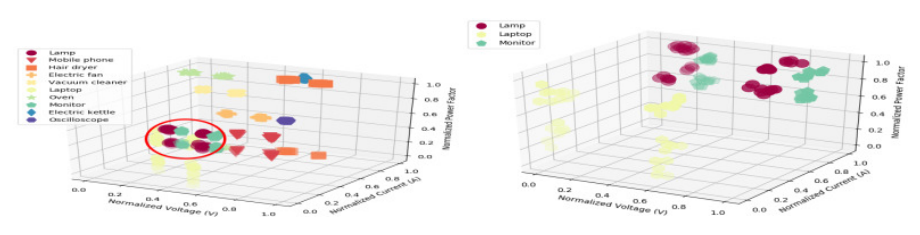

Figure 6. (a) Different appliances with similar load signatures;

(b) Retain the unrecognized appliances and cluster with multiple granularities

\section{Prototyping And Experimental Studies}

In this section, the prototype of the smart socket is implemented, including the hardware components and the method of data transmission. Furthermore, to validate the performance of our proposed recursive DBSCAN approach for appliances recognition, two experiments about the selection of Eps values and the effectiveness of recursion are demonstrated. We develop corresponding programs using Python language. Our programs are run on a PC with Intel i73770, 3.4GHz CPU and 24GBytes RAM to implement the approach. 


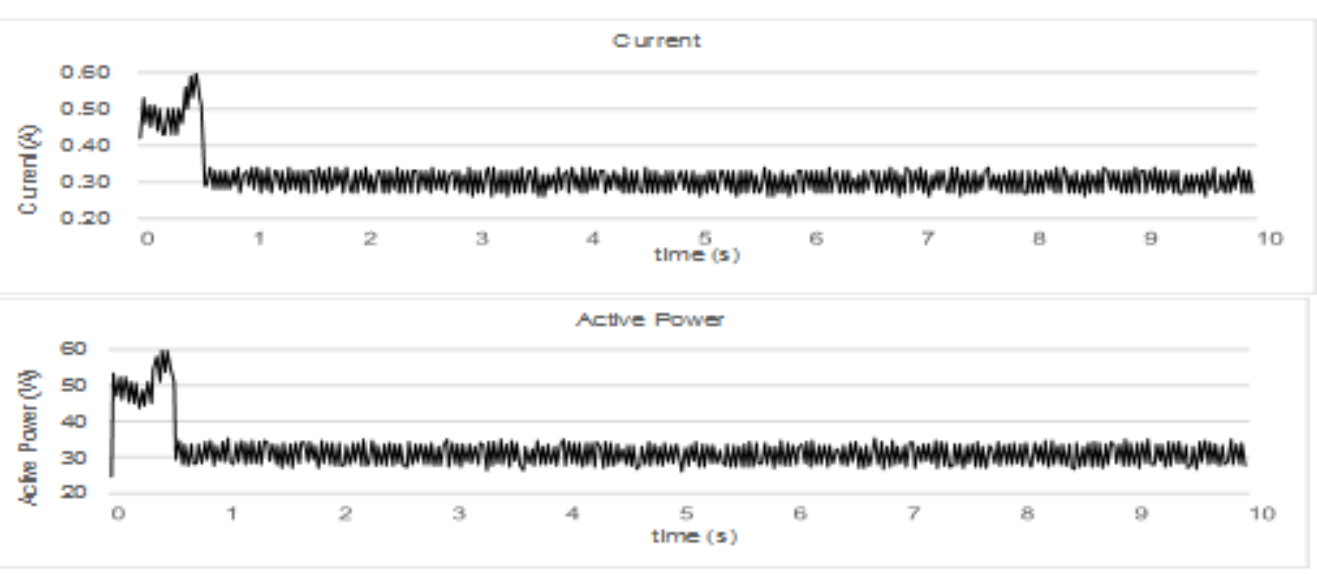

Figure 7. Current and active power collected by our smart socket

A total of 10 different household appliances are used in this study for experimental analysis including lamp, mobile phone, hair dryer, electric fan, vacuum cleaner, laptop, oven, monitor, electric kettle and oscilloscope. The smart socket records load signatures with a frequency of 40 $\mathrm{Hz}$ including voltages $(\mathrm{V})$, currents $(\mathrm{I})$, power factor $(\mathrm{PF})$, active power $(\mathrm{P})$, reactive power $(\mathrm{Q})$ and apparent power $(\mathrm{S})$ as shown in Fig. 7. Those data samples are saved ordered by collecting time as shown in table 1 . When the system starts the process of recognition, the data samples may be normalized and mapped into the feature space as shown in Fig. 8. Each node in the feature space represents a data samples.

Table 1. Data samples collected in our scheme

\begin{tabular}{|r|r|r|r|r|r|r|}
\hline Timestamp & $\begin{array}{c}\text { Voltage } \\
(\mathrm{V})\end{array}$ & $\begin{array}{c}\text { Current } \\
(\mathrm{A})\end{array}$ & $\begin{array}{c}\text { Power } \\
\text { Factor }\end{array}$ & $\begin{array}{c}\text { Active } \\
\text { Power(P) }\end{array}$ & $\begin{array}{c}\text { Apparent } \\
\text { Power(VA) }\end{array}$ & $\begin{array}{c}\text { Reactive } \\
\text { Power(VAR) }\end{array}$ \\
\hline 15:03:45.635 & 113.04 & 0.42 & 0.434 & 24.7 & 25.71 & 7.14 \\
\hline 15:03:45.650 & 109.89 & 0.53 & 0.669 & 53.56 & 50.98 & 21.09 \\
\hline $15: 03: 45.676$ & 112.81 & 0.46 & 0.589 & 47.23 & 57.35 & 20.87 \\
\hline $15: 03: 45.690$ & 110.02 & 0.51 & 0.676 & 52.53 & 49.88 & 20.1 \\
\hline $15: 03: 45.717$ & 113.12 & 0.45 & 0.572 & 46.17 & 55.59 & 20.54 \\
\hline $15: 03: 45.733$ & 110.02 & 0.51 & 0.672 & 52.47 & 49.66 & 19.89 \\
\hline $15: 03: 45.758$ & 109.89 & 0.44 & 0.571 & 45.31 & 55.37 & 20.32 \\
\hline $15: 03: 45.800$ & 112.53 & 0.5 & 0.693 & 51.06 & 48.34 & 19.56 \\
\hline $15: 03: 45.815$ & 109.67 & 0.43 & 0.599 & 44.93 & 54.49 & 20.1 \\
\hline $15: 03: 45.841$ & 112.9 & 0.43 & 0.668 & 50.6 & 54.49 & 19.12 \\
\hline
\end{tabular}

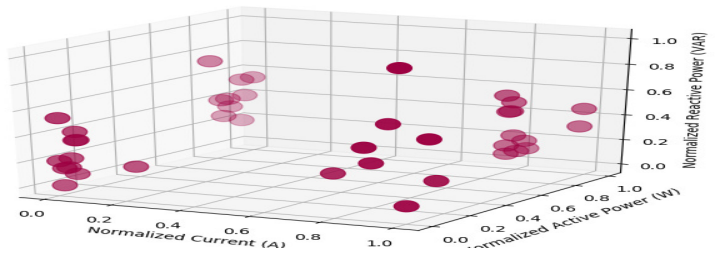

Figure 8.Mapping the data samples into the feature space.

\subsection{HARDWARE IMPLEMENTATION OF THE SMART SOCKET}

To obtain electrical power characteristics of electrical devices and control them, we develop the smart socket, which is cost-reduction, and its size is small enough to be deployed as standard 
sockets. We have implemented a smart socket, as shown in Fig.9. The smart socket consists of three modules:

\section{1) Sensor Module}

A sensor module is used to measure the current and voltage signal. Among the many alternatives, we select a Hall Effect current sensor ACS71020 because it includes Analog-to-Digital Converter (ADC) and supports Serial Peripheral Interface Bus (SPI) which is hardware communication protocol used for two-way communication between the sensor module and control module.

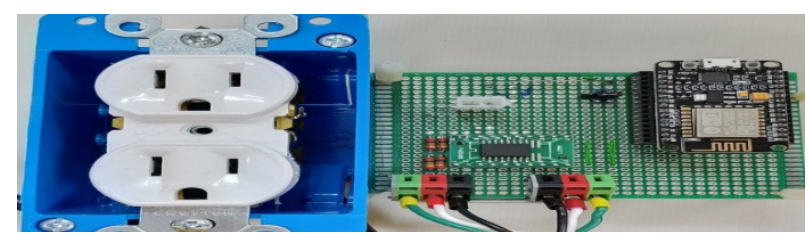

Figure 9. Smart socket designed prototype

\section{2) Control Module}

We select ESP8266 as our microcontroller. It features a small size with a Wi-Fi chip, low power consumption, and compatible with many development environments. Its main functions are processing signals, storing it in its memory, and publishing data to MQTT broker.

\section{3) Communication Module}

The communication module is needed to transfer the data published by the control module. We use Wi-Fi to upload data to the MQTT broker because of its coverage range. MQTT is a straight forward simple and lightweight messaging protocol so that it doesn't contain redundant information to occupy bandwidth and waste memory space.

\subsection{EXPERIMENT \#1: USING Different PARAMETER SETTINGS In DBSCAN}

In Experiment \#1, in order to compare the recognition effectiveness of different Eps values setting, we randomly plug each appliance into the smart socket and turn it on for 5 seconds.

We set the Eps value from 0.3 to 0.6 in DBSCAN and try to recognize the 10 different appliances by turns. Figure 10 shows the clustering performance of different Eps values setting. The result shows that Eps $=0.3$ corresponds to an accuracy of $60 \%$. When the Eps value is smaller than 0.3, the performance of DBSCAN approach is not effective enough because a lot portion of the data samples are regarded as the noise and construct a few clusters. By this experiment, we learn that changing the parameters of DBSCAN is insufficient to recognize all of the appliances. Therefore, we implement recursive DBSCAN with multiple granularities in Experiment \#2. 

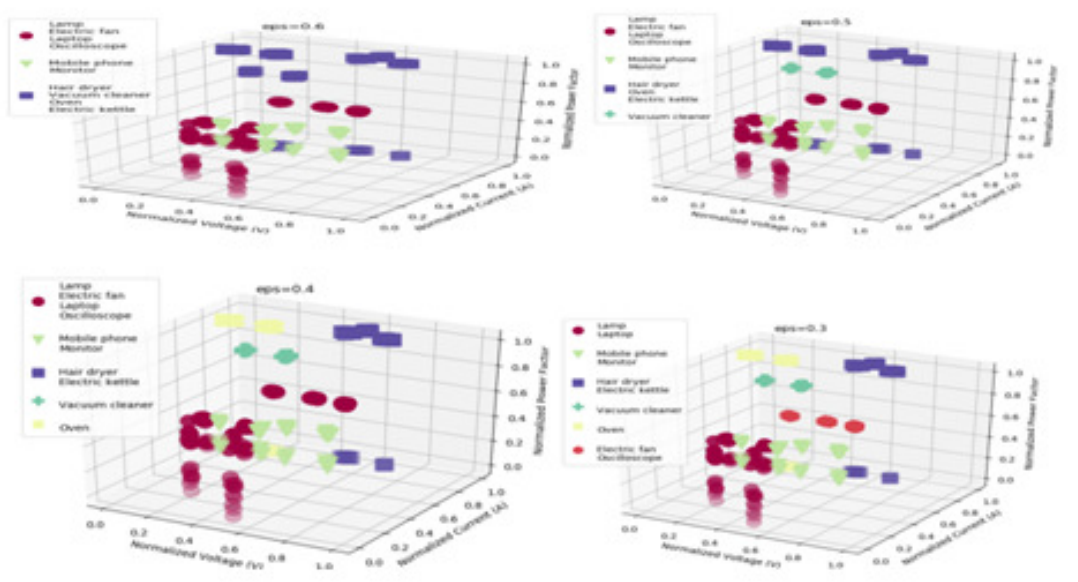

Figure 10 . Clustering results with different parameter settings

\subsection{EXPERIMENT \#2: RECURSIVE DBSCAN With MULTIPLE GRANULARITIES}

In order to recognize the appliances more accurate, in Experiment \#2, we demonstrate the proposed recursive DBSCAN with multiple granularities. The recognition result of different recursion times and the number of appliances is shown in Fig. 11. Note that Recursive(2) means that the DBSCAN algorithm is recursively conducted for two more times.

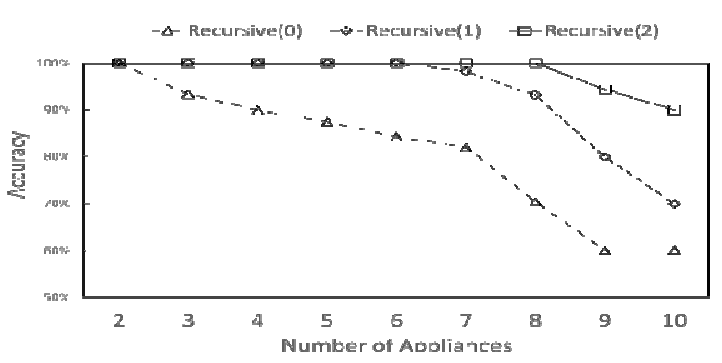

Figure 11. Impact of the proposed recursive DBSCAN

The accuracy of the first recursion for 10 appliances is $60 \%$, and then we retain the appliances that have not been recognized and implement the DBSCAN again for the second recursion. Eventually, the result shows that after three recursions of DBSCAN, the accuracy of 10 appliances recognition increase to $90 \%$. In this case, the only appliances that cannot be recognized clearly are hair dryer and electric kettle because their load signatures are quite similar. When the system detects the appliances are turning on and make an incorrect recognition learning from user feedback, our system recursively implements the DBSCAN with multiple granularities until the appliances can be precisely recognized.

\subsection{USer Interface Of OUR Proposed Scheme}

To display the performance and present the information of the electricity consumption, we have produced a user interface, as shown in Fig.12.User can interact with our system in some of the scenarios. For example, our system can notify users when an appliance has already operated for a long time and consumed power exceedingly. Fig.12 (a) shows the information of the appliances operating time and the used energy above the interface, the system records the power consumption and operating time since the appliance plugged in and recognized. Moreover, realtime power proportion of operating appliances in the smart grid can also be shown in the user 
interface as a doughnut chart. Through this function in the user interface, the report of the consumption record in a period of time can provide a more efficient review for users. Figure 12(b) shows that when the system detects an appliance that has not been recorded, a dialog box will emerge in the interface for requesting a name to the appliance. Users can respond their feedback if the system makes an inaccurate recognition. As mentioned above, our system can implement the proposed recursive DBSCAN with multiple granularities

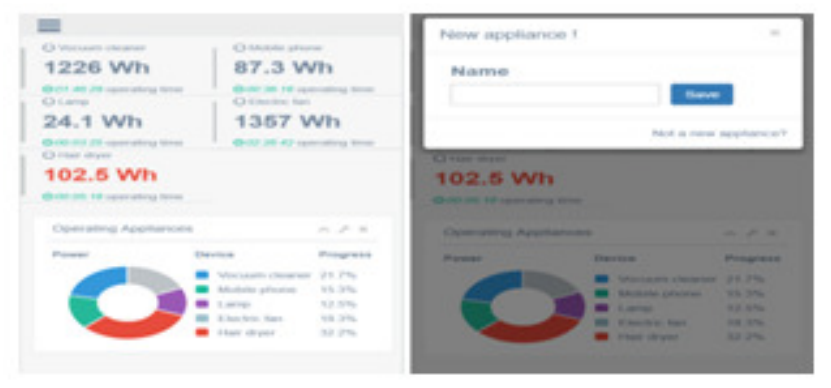

Figure 12. User interface: (a) Real-time appliances information

(b) Dialog box for user feedback

\section{Conclusions}

In this work, we have addressed the problem of equipping smart sockets into the smart grid with extensive installation constraint. To tackle the problem, we have proposed an appliance recognition approach to realize a smart socket that can distinguish several appliances .In the practical application scenario, the prior knowledge of the new appliances cannot be learned, so the classification approaches are not suitable for the appliances recognition task. Our proposed recognition approach is based on DBSCAN. However, existing DBSCAN is not suitable for the scenario because the difference of load features between appliances are inconsistent. For better effectiveness, we have further proposed a new approach that combines existing DBSCAN with recursion for an accurate solution. The experiments in practice have showed the effectiveness of proposed recursive DBSCAN. The accuracy increases from $60 \%$ to $90 \%$ after 3 times of recursion.

\section{REFERENCES}

[1] k. Ma, c. Wang, j. Yang, z. Tian, and x. Guan, (2017) "energy management based on demand-side pricing: a super modular game approach",ieee access, vol. 5, pp.18219-18228.

[2] m. Li, w. Gu, w. Chen, y. He, y. Wu, and y. Zhang, (2018) "smart home: architecture, technologies and systems",procedia computer science, vol. 131, pp.393-400.

[3] p. Palensky, d. Dietrich, (2011) "demand side management: demand response intelligent energy systems and smart loads", ieee trans. Ind. Inform., vol. 7, no. 3, pp.381-388.

[4] q.yan, y. Jiancheng, k.xiangyu, w. Xudong, m. Yuying, and 1. Bo, (2018) "home smart energy network configuration considerations for demand response", ieeein 2018 china international conference on electricity distribution, pp. 2875-2879.

[5] d.m. han, j.h. lim, (2010) "smart home energy management system using ieee 802.15 .4 and zigbee communication", ieee trans. On consumer electronics, vol. 56, no. 3, pp.1403-1410.

[6] óblanco-novoa,t. Fernández-caramés, p. Fraga-lamas, andl. Castedo, (2017)“an electricity price-aware open-source smart socket for the internet of energy", sensors,vol. 17, no. 3,pp.643. 
[7] a. Ridi, c. Gisler, and j. Hennebert, (2014) "a survey on intrusive load monitoring for appliance recognition", ieee proceedings of the 22 nd international conference on pattern recognition,pp.37023707 .

[8] a. Zoha, a. Gluhak, m. Imran, and s. Rajasegarar, (2012) "non-intrusive load monitoring approaches for disaggregated energy sensing: a survey”, sensors, vol. 12, no.12 pp.16838-16866.

[9] i. Abubakar, s. N. Khalid, m. W. Mustafa, h. Shareef, andm. Mustapha, (2017). "application of load monitoring in appliances' energy management-a review",renewable and sustainable energy reviews, vol. 67 , pp.235-245.

[10] h. H.chang, c. L. Lin, and h. T. Yang, (2008)"load recognition for different loads with the same real power and reactive power in a non-intrusive load-monitoring system", international conference on computer supported cooperative work in design, pp.1122-1127.

[11] s. Kong, y. Kim, r. Ko, and s. K. Joo, (2015) "home appliance load disaggregation using cepstrumsmoothing-based method", ieee transactions on consumer electronics,vol. 61, no. 1, pp.24-30.

[12] h. Y. Lam, g. S. K. Fung, and w. K. Lee, (2007) "a novel method to construct taxonomy electrical appliances based on load signatures", ieee transactions on consumer electronics, vol. 53, no.2, pp.653660.

[13] p. Meehan, s. Phelan, c. Mcardle, and s. Daniels, (2012) "temporal and frequency analysis of power signatures for common household appliances", iet proceedings of the symposium on ict and energy efficiency and workshop on information theory and security, pp.22-27.

[14] v. Abeykoon, n. Kankanamdurage, a. Senevirathna, p. Ranaweera. And r. Udawalpola,(2016) "real time identification of electrical devices through power consumption pattern detection", proceedings of the international conference on micro and nano technologies, modelling and simulation, pp.1-3.

[15] h. P.kriegel,p. Kröger,j. Sander,anda. Zimek, (2011) “density-based clustering”, wiley interdisciplinary reviews: data mining and knowledge discovery, vol. 1, no. 3, pp.231-240.

[16] m.ankerst,m. M. Breunig,h. P.kriegel, and j. Sander, (1999) "optics: ordering points to identify the clustering structure" acm.in acm sigmod record, vol. 28, no. 2, pp.49-60.

[17] m. Ester, h. P. Kriegel, j. Sander, and x. Xu, (1996)"a density-based algorithm for discovering clusters in large spatial databases with noise",proceedings of the second international conference on knowledge discovery and data mining, pp.226-231. 